\title{
The importance of sonographic evaluation of muscle depth and thickness prior to the 'tiny percutaneous needle biopsy'
}

\author{
Tereza Jandova (1), Danilo Bondi (1), Vittore Verratti (2), Marco Narici (3), Michal
} Steffl, (4) Tiziana Pietrangelo (1)

(1) Department of Neuroscience, Imaging and Clinical Sciences, University” G. d'Annunzio" of Chieti - Pescara, Italy (2) Department of Psychological, Health and Territorial Sciences, University "G. d'Annunzio” of Chieti - Pescara, Italy (3) Department of Biomedical Sciences, University of Padova, Italy (4) Faculty of Physical Education and Sport, Charles University, Prague, Czech Republic

This article is distributed under the terms of the Creative Commons Attribution Noncommercial License (CC BY-NC 4.0) which permits any noncommercial use, distribution, and reproduction in any medium, provided the original author(s) and source are credited.

\begin{abstract}
Biopsy of human skeletal muscle tissue is a widely used method in many research studies, where 'the tiny percutaneous needle biopsy' (TPNB) is one of the relatively simplest and safest procedures currently available. By using and contrasting ultrasound images of vastus lateralis of young and elderly subjects, this work highlights further the safety aspects of TPNB and stresses the importance of prior ultrasound evaluation of muscle depth and thickness in order to prevent wrong muscle group or tissue sampling in subsequent laboratory analyses.
\end{abstract}

Key Words: muscle imaging, echography, vastus lateralis, muscle sample, ultrasound

Eur J Transl Myol 30 (1): xx1-xx5, 2020

Needle biopsy of human skeletal muscle tissue is a widely used method in many research studies in order to investigate the cellular and molecular aspects of skeletal muscle under different conditions including ageing, ${ }^{1}$ exercise, ${ }^{2}$ or disease states. ${ }^{3}$ Particularly useful in this respect is the 'tiny percutaneous needle biopsy' (TPNB) that without compromising the quality of the myofibre samples has proved to be a less invasive, easier and a safer procedure than the traditional conchotome, ${ }^{4}$ or the needle aspiration biopsy (see for more details Pietrangelo et al. ${ }^{5,6}$ ). However, to identify and interpret the cellular and molecular aspects of the muscle tissue of interest at best, good quality muscle biopsy specimens ought to be obtained before any subsequent laboratory analyses.
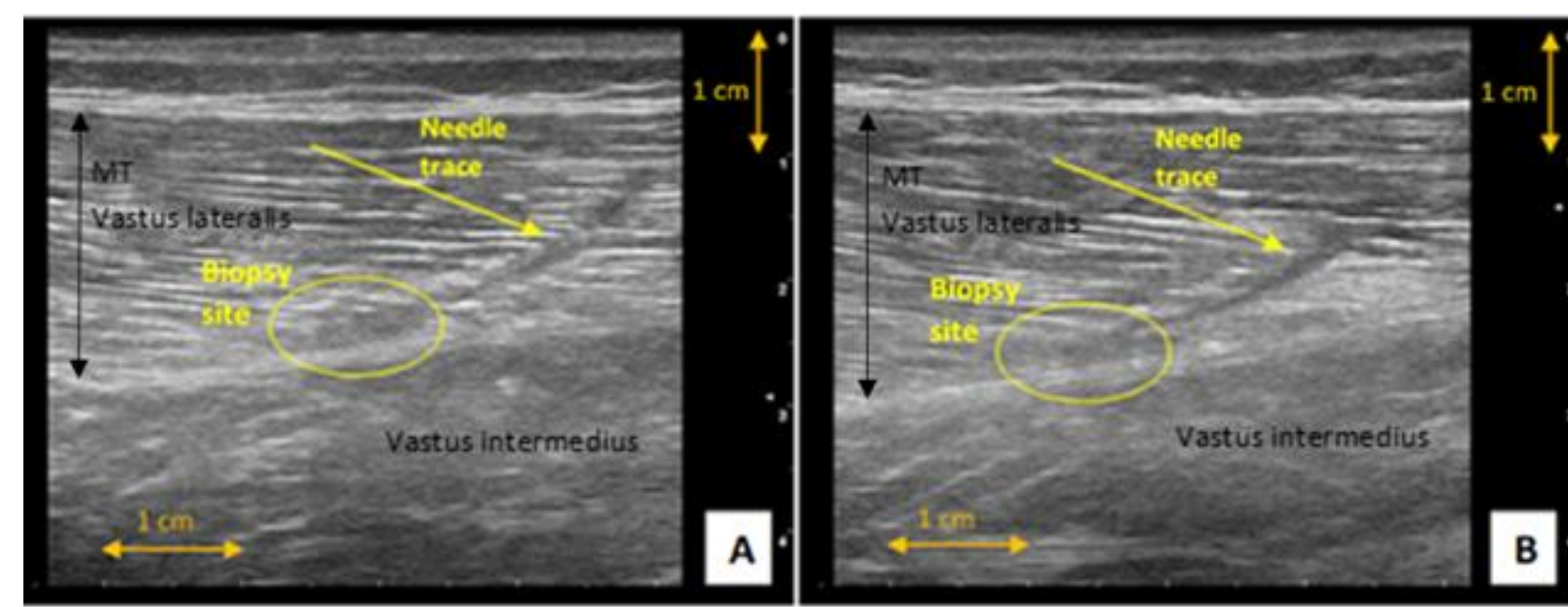

Fig 1. Ultrasound images of vastus lateralis, of a 32 years-old male subjects without any acute or chronic muscular disease (BMI: $23.1 \mathrm{Kg} / \mathrm{m}^{2}$, estimated Fat-Free Mass from bioimpedance: 80\%); A-first day after biopsy, $B$ fourth day after biopsy; images from "Kanchenjunga Exploration \& Physiology" research project 


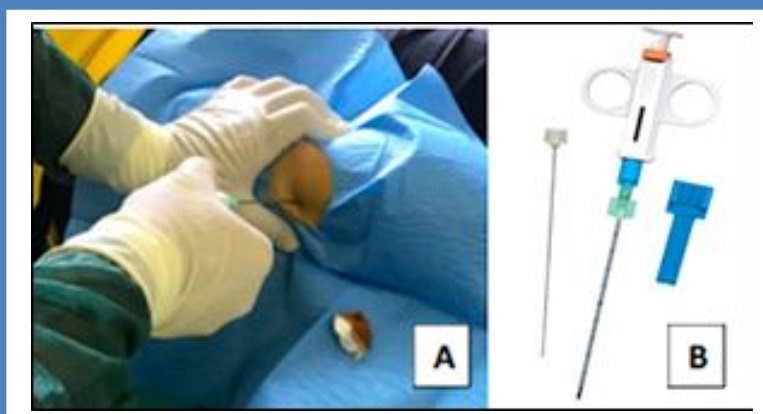

Fig 2.. Tiny percutaneous needle biopsy from vastus lateralis muscle; A - Sterile procedure during muscle biopsy at a one-third distal distance between the greater trochanter and patellar top border performed by a trained medical doctor; B - Semi-automatic needle used for the biopsy (PRECISA® model 1310 - HS Hospital Service $S p A$, Latina, Italy)

Sonographic guidance for the prior assessment of muscle thickness (MT) or site of sampling has been already recommended previously; however, it is still too rarely performed. ${ }^{7,8}$ This work intends to further highlight the importance of ultrasound evaluation of muscle depth (MD) and MT prior to muscle biopsy collection and reports on the potential risks of obtaining different or inadequate muscle biopsy samples. . The ultrasound (US) images reported in this paper were acquired using longitudinal B-mode US scanning of the VL at the distal one-third of the length between the greater trochanter and the patellar top border, acquired with a 5-cm, 3-11 MHz linear array probe (Mylab Gamma, Esaote Biomedica, Genova, Italy). MT of VL was marked by either a black or white arrow (depending on the echogenicity of muscles) as a distance from superficial to deep aponeurosis. The aponeuroses appear as brightly, whitish-silvery colour, echogenic linear structures on the US images. MD is marked by a vertical yellow arrow.

Figure 1 shows two US images of VL of 32 years old male taken at $1^{\text {st }}$ and $4^{\text {th }}$ day after TPNB. A clear needle trace and the site of biopsy is visible in both images. As the figure shows, there is no evidence of oedema in both images, and the needle trace seems to be visibly reduced on the day $4^{\text {th }}$ (panel B) compared to the day $1^{\text {st }}$ (panel A), suggesting a rapid and successful muscle wound healing process. Although TPNB is classed as a minor surgical procedure, the acute muscle damage is very minimal, usually resulting only in slight soreness or discomfort lasting max. 1-3 days. Therefore, healthcare providers should recommend restricting any intense physical activity for $24-$ to- $48 \mathrm{~h}$ post-biopsy. This makes the TPNB as one of the safest and the least invasive muscle biopsy technique currently available.

By using longitudinal ultrasound images of the vastus lateralis (VL) muscle obtained from three male subjects who participated in a recent research project "Kanchenjunga Exploration \& Physiology", where one of the research methods included muscle biopsy from (VL) using the TPNB technique (Fig 2), we could demonstrate the importance of performing a sonographic evaluation of MD and MT before taking muscle biopsy samples. Instead, Fig shows two US images of VL of the other two male subjects who participated in the study, detailing different muscle morphology. As may be observed in these images, each subject exhibited different MT ( $\sim \mathrm{cm}$ vs. nearly $3 \mathrm{~cm}$ ), which resulted in the biopsy specimens being taken from slightly different sites. The biopsy specimens from the first subject (48 years old) were taken at the border of the deep aponeurosis (panel A), whereas the biopsy samples from the second subject (25 years old) were taken from the middle of the VL
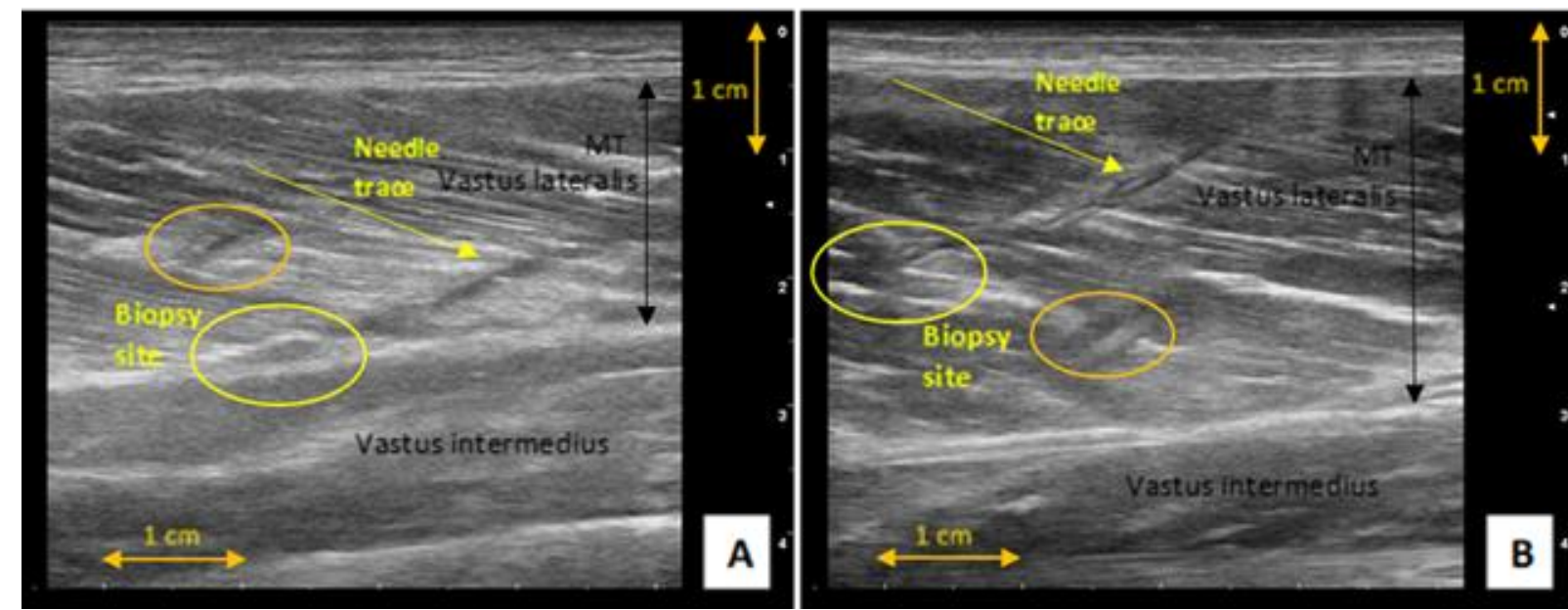

Fig 3.. Ultrasound images of vastus lateralis; the orange circle shows a biopsy site after 7 weeks (pre-Nepal); A 48 years-old male subjects without any acute or chronic muscular disease (BMI: $28.0 \mathrm{Kg} / \mathrm{m}^{2}$, estimated FatFree Mass from bioimpedance: 77\%); B - 25 years-old male subjects without any acute or chronic muscular disease (BMI: $23.1 \mathrm{Kg} / \mathrm{m}^{2}$, estimated Fat-Free Mass from bioimpedance: 79\%); images from "Kanchenjunga Exploration \& Physiology" research project 

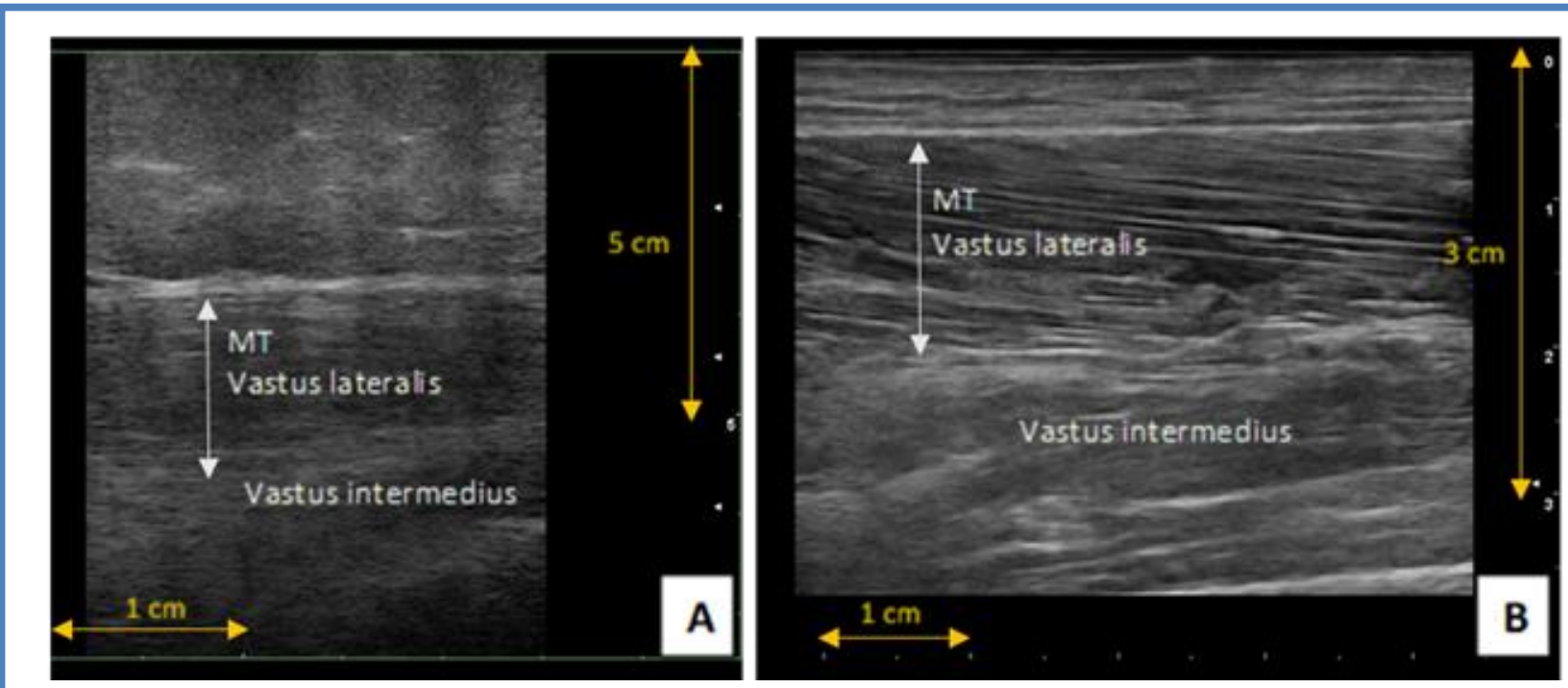

Fig 4.. Ultrasound images of vastus lateralis of sarcopenic elderly subjects; A - 83 years old sarcopenic obese female; $B$ - 84 years old sarcopenic male

muscle (panel B). Such findings are of laboratory importance as by taking the aponeurosis specimens together with the muscle any subsequent laboratory analyses could be affected, likely leading to some 'skewed' conclusions. In the previous subject, the biopsy samples were also taken near the deep aponeurosis (see Fig 1). It is, therefore, fundamental that prior US evaluation of MT and MD is performed. Fig 3 details also the biopsy site (orange circles in the images) after 7 weeks of TPNB with no visible needle traces, suggesting successful regeneration of muscle tissue without the formation of a connective tissue scar.

Our findings are particularly important when considering the studies involving elderly individuals. Currently, many researchers are interested in identifying the exact cellular and molecular mechanisms behind sarcopenia using muscle biopsy samples in their laboratory investigations. ${ }^{9-11}$

With this respect, TPNB seems to be the most suitable muscle biopsy technique for the elderly. However, one should also recognise the fact that the elderly population is very heterogeneous, ranging from healthy active individuals to obese or sarcopenic; thus, it is of crucial importance to evaluate their muscles beforehand. For illustration, we present a selection of US images, detailing the heterogeneity in muscle morphology in the elderly population.

Fig 4 shows US images of VL of two sarcopenic and Fig 5 of two healthy active elderly individuals. As seen from the images, the MD and MT are very different in all the elderly individuals. In terms of the sarcopenic ones, the female's VL is about $4-5 \mathrm{~cm}$ in-depth, under a prominent $\sim 3 \mathrm{~cm}$ layer of subcutaneous tissue (Fig $4, \mathrm{~A}$ ), whereas
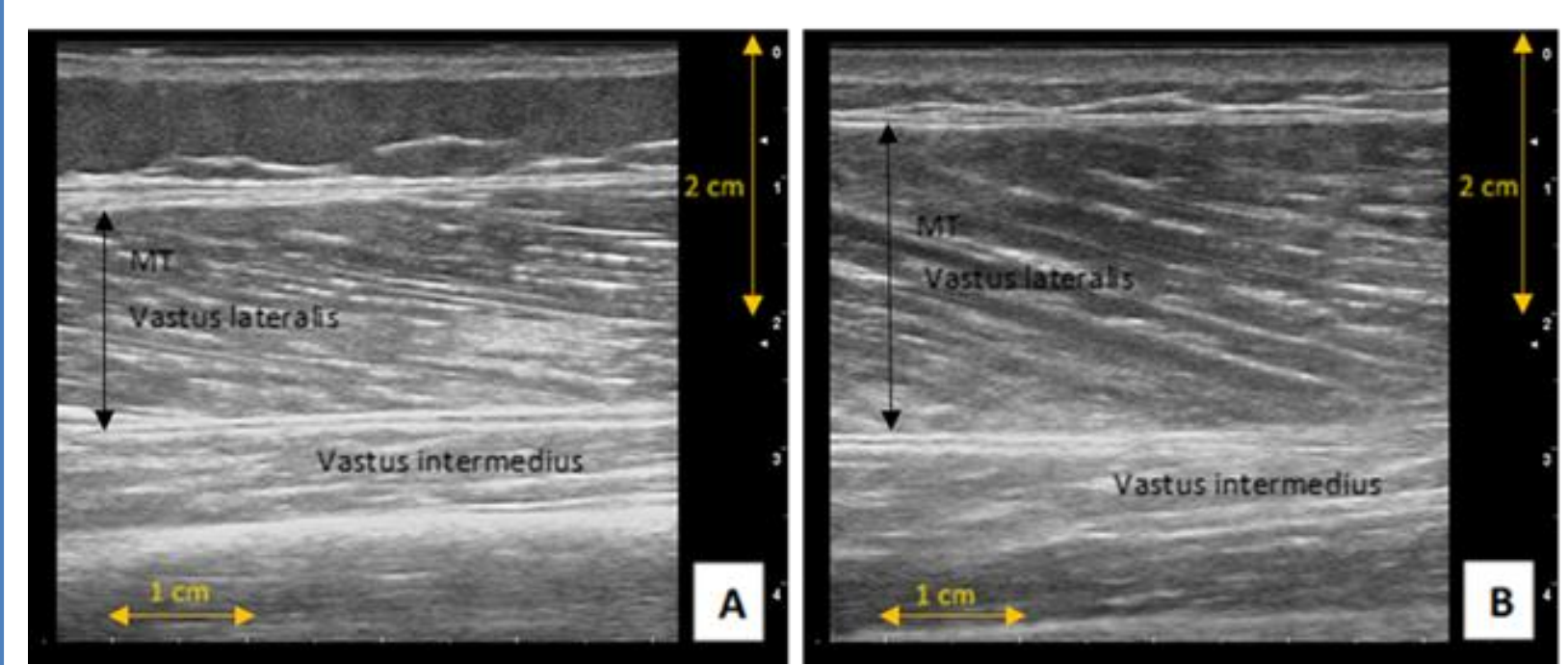

Fig 5. Ultrasound images of vastus lateralis at a one-third distal distance between great trochanter and patellar top border of healthy elderly subjects; A) 66 years old female; B) 68 years old male 
the sarcopenic male (panel B) has virtually no subcutaneous tissue and his VL is no more than $2 \mathrm{~cm}$ indepth. A similar trend, however not so prominent, is visible in the healthy active female and male individuals, where the female has a small layer of subcutaneous tissue and smaller MT (Fig 5, A) than the male (panel B). Thus, extra caution must be taken before obtaining muscle biopsy samples from this population in order to prevent the wrong muscle group or tissue sampling. In fact, US has already been proposed as a useful tool for clinicians as a non-invasive, inexpensive and objective measurement of muscle mass and architecture in geriatrics, ${ }^{12,13}$ and it can also serve as a highly useful prior mean in the assistance to optimal muscle needle biopsies These observations seem to warrant the use of the TPNB as one of the safest and minimally invasive biopsy techniques currently available. In addition, prior evaluation of MD and MT using ultrasound seems a highly useful strategy for obtaining good quality muscle biopsy tissue while using a minimally invasive surgical approach, avoiding the risk of having incorrect muscle biopsy specimens.

\section{List of acronyms}

TPNB - Tiny Percutaneous Needle Biopsy

MT; Muscle Thickness

MD - Muscle Depth

VL - Vastus Lateralis

US - ultrasound

\section{Authors contributions}

TJ, DB, VV, MN and TP designed the work; TJ, DB, VV and TP did the analyses; all the authors drafted, revised and approved the manuscript.

\section{Acknowledgments}

We would like to thank all the volunteers who participated in the study.

\section{Funding}

The study was funded by: the "G. d'Annunzio" University grants to TP and VV; the "Departments of Excellence 2018-2022" initiative of the Italian Ministry of Education, University and Research to the Department of Neuroscience, Imaging and Clinical Sciences of "G. d'Annunzio" University, Italy; the project Q41 from the Charles University, Czech Republic to MS.

\section{Conflict of Interest}

The authors declare they have no financial, personal, or other conflicts of interest.

\section{Ethical Publication Statement}

We confirm that we have read the Journal's position on issues involved in ethical publication and affirm that this report is consistent with those guidelines. The study was approved by the Ethics Committee of "G. d'Annunzio" University (protocol n. 16/2019) and was in accordance to the Declaration of Helsinki..

\section{Corresponding Author}

Tereza Jandova, Department of Neuroscience, Imaging and Clinical Sciences, University "G. d'Annunzio" of Chieti - Pescara, Via dei Vestini, 31, 66100 Chieti, Italy. ORCID iD: 0000-0002-5603-5670

Email: te.jandova@hotmail.com

E-mails of co-authors

Danilo Bondi: danilo.bondi@unich.it

ORCID iD: 0000-0003-1911-3606

Vittore Verratti: vittore.verratti@unich.it

ORCID iD: 0000-0001-8343-9024

Marco Narici: marco.narici@unipd.it

ORCID iD: 0000-0003-0167-1845

Michal Steffl: steffl@ftvs.cuni.cz

ORCID iD: 0000-0001-7297-8145

Tiziana Pietrangelo: tiziana.pietrangelo@unich.it

ORCID iD: 0000-0002-7507-1255

\section{References}

1. Dodds RM, Davies K, Granic A, et al. Mitochondrial respiratory chain function and content are preserved in the skeletal muscle of active very old men and women. Exp Gerontol 2018;113:80-5.

2. Franchi MV, Atherton PJ, Reeves ND, et al. Architectural, functional and molecular responses to concentric and eccentric loading in human skeletal muscle. Acta Physiol (Oxf) 2014;210:642-54.

3. Wagner KR, Fleckenstein JL, Amato AA, et al. A phase I/IItrial of MYO-029 in adult subjects with muscular dystrophy. Ann Neurol 2008;63:561-71.

4. Dietrichson P, Coakley J, Smith PE, et al. Conchotome and needle percutaneous biopsy of skeletal muscle. J Neurol Neurosurg Psychiatry 1987;50:1461-7.

5. Pietrangelo T, D'Amelio L, Doria C, et al. Tiny percutaneous needle biopsy: An efficient method for studying cellular and molecular aspects of skeletal muscle in humans. Int $\mathrm{J}$ Mol Med 2011;27:361-7.

6. Pietrangelo T, Perni S, Di Tano G, et al. A method for the ultrastructural preservation of tiny percutaneous needle biopsy material from skeletal muscle. Int J Mol Med 2013;32:965-70.

7. O'Sullivan PJ, Gorman GM, Hardiman OM, et al. Sonographically guided percutaneous muscle biopsy in diagnosis of neuromuscular disease: a useful alternative to open surgical biopsy. J Ultrasound Med 2006;25:1-6.

8. Wilson D, Breen L, Lord JM, Sapey E. The challenges of muscle biopsy in a community based geriatric population. BMC Res Notes 2018;26:11:830.

9. Ryall JG, Schertzer JD, Lynch GS. Cellular and molecular mechanisms underlying age-related skeletal muscle wasting and weakness. Biogerontology 2008;9:213-28. 
10. Welle S. Cellular and molecular basis of age-related sarcopenia. Can J Appl Physiol 2002;27:19-41.

11. Verdijk LB, Dirks ML, Snijders T, et al. Reduced satellite cell numbers with spinal cord injury and aging in humans. Med Sci Sports Exerc 2012;44:2322-30.

12. Ticinesi A, Narici MV, Lauretani F, et al. Assessing sarcopenia with vastus lateralis muscle ultrasound: an operative protocol. Aging Clin Exp Res 2018;30:1437-43.
13. Kemp GJ, Birrell F, Clegg PD, et al. Developing a toolkit for the assessment and monitoring of musculoskeletal ageing. Age and Ageing 2018;47:iv1-iv19.

Submission: January, 27, 2020

Acceptance: January 28, 2020 